\title{
Interactions microorganismes - matériaux de construction
}

\author{
A. Bertron ${ }^{1}$, F. Feugeas ${ }^{2}$ et I. Frateur ${ }^{3}$
}

Reçu le 27 avril 2015, accepté le 30 avril 2015

\begin{abstract}
Résumé - Les interactions entre les microorganismes et les matériaux de construction dans leur environnement influencent la durabilité des ouvrages, la santé et le confort dans les bâtiments ainsi que les qualités esthétiques des ouvrages. Les impacts économiques, sociétaux et environnementaux de ces phénomènes sont majeurs. Les articles présentés dans ce numéro, issus des contributions au Forum Jeunes Chercheurs, organisé par la Commission Biodétérioration des Matériaux du CEFRACOR, montrent un échantillon significatif des recherches réalisées en France dans les différentes thématiques relatives aux interactions microorganismes-matériaux.
\end{abstract}

Mots clés : Microorganismes / matériaux de construction / biodétérioration

Abstract - Microorganisms-building materials interactions. Issues related to the interactions between microorganisms and building materials in their environment, influence the sustainability of the structures, health and comfort in the buildings and the aesthetic qualities of the structures. Economic, societal and environmental impacts of these phenomena are major. The articles in this issue, coming from the contributions to the Forum of Young Researchers, organized by the "Biodeterioration of Materials" Committee of CEFRACOR, represent a significant sample of the research carried out in France in different topics related to the microorganisms-materials interactions.

Key words: Microorganisms / building materials / biodeterioration

Les problématiques liées aux interactions entre les microorganismes et les matériaux de construction dans leur environnement sont présentes dans différents contextes du BTP. Elles influencent la durabilité des ouvrages (réseaux d'assainissement [1], ouvrages hydroélectriques en eaux douces [2], structures agro-industrielles, ouvrages de méthanisation [3], installations portuaires, ouvrages de traitement des eaux usées...), la santé et le confort dans les bâtiments [4] (qualité de l'air dégradée par les proliférations microbiennes) ainsi que les qualités esthétiques des ouvrages (prolifération d'algues sur les parements). Les interactions bio-géo-chimiques dans les ouvrages de stockage profond des déchets radioactifs sont également susceptibles de diminuer le niveau de sécurité des enfouissements [5]. Les impacts économiques, sociétaux et environnementaux de ces phénomènes sont majeurs.

En contrepartie, les microorganismes peuvent, de manière directe ou indirecte, avoir un effet bénéfique sur les matériaux de construction. Par exemple, des technologies de bio-cicatrisation des matériaux cimentaires sont développées pour colmater les micro-fissures dans l'objectif d'améliorer leurs propriétés de transfert et donc la durabilité de ces matériaux. Des produits bio-sourcés sont également développés à partir de composés issus de la

1 Maître de Conférences HDR, IUT Toulouse A, LMDC, Membre de la Commission Biodétérioration des Matériaux du CEFRACOR et éditrice invitée de Matériaux et Techniques, Laboratoire Matériaux et Durabilité des Constructions, Université de Toulouse, INSA/UPS Dép. Génie Civil, 135 avenue de Rangueil, 31077 Toulouse Cedex 4, France

2 Présidente de la Commission Biodétérioration des Matériaux du CEFRACOR, Professeur des Universités, INSA de Strasbourg, Laboratoire ICube, 24 boulevard de la Victoire, 67084 Strasbourg Cedex, France

3 Chargée de Recherche HDR CNRS, Secrétaire de la Commission Biodétérioration des Matériaux du CEFRACOR, Laboratoire Interfaces et Systèmes Electrochimiques (LISE), UMR 8235 CNRS-UPMC, Université Pierre et Marie Curie, Case 133, 4 place Jussieu, 75252 Paris Cedex 05, France 
matrice extracellulaire de certaines bactéries et permettent de limiter la biocontamination de la surface des bétons armés et d'améliorer la résistance à la corrosion de leurs armatures métalliques [6,7].

Un certain nombre d'équipes de chercheurs travaillent aujourd'hui en France sur ces thématiques. Elles s'attachent notamment à étudier les mécanismes d'interactions entre microorganismes et matériaux de construction, à caractériser les performances des matériaux dans ces environnements et à développer des méthodes innovantes et pertinentes pour ces caractérisations $[1,2,6,8]$. L'objectif ultime de ces travaux est généralement d'améliorer les performances des matériaux de construction et des ouvrages dans leur environnement.

La Commission Biodétérioration des Matériaux du Centre Français de l'Anticorrosion (CEFRACOR) a pour vocation de rassembler la communauté interdisciplinaire des acteurs académiques et industriels préoccupés par les problèmes de biodétérioration et de créer une plateforme d'échanges à l'échelle nationale. En sus de ses réunions régulières, la Commission organise ou participe activement à des Ecoles Thématiques CNRS. Par ailleurs, la Commission organise environ tous les 18 mois un Forum Jeunes Chercheurs. La dernière édition de ce forum a eu lieu dans le cadre de l'École Thématique BIODEMAT 2014 «Biodétérioration des matériaux : interactions biofilms-alliages métalliques ou bétons $»$ qui s'est tenue en octobre 2014 à La Rochelle. À l'issue de ce XII ème Forum et dans le cadre de l'accord entre le CEFRACOR et la revue Matériaux \& Techniques, les textes des communications ont été rédigés par les jeunes chercheurs participants et, après évaluation par un binôme d'experts, les papiers acceptés, font l'objet de ce numéro thématique. Les articles présentés ici montrent ainsi un échantillon, certes non exhaustif, mais significatif des recherches réalisées en France dans les différentes thématiques relatives aux interactions microorganismes-matériaux.

\section{Références}

[1] M. Peyre Lavigne, A. Bertron, C. Patapy, X. Lefebvre, E. Paul, Matériaux \& Techniques 103 (2015) 204

[2] K. Ben Ahmed, N. Serres, C. Fond, F. Feugeas, Matériaux E Techniques 103 (2015) 203

[3] C. Voegel, A. Bertron, B. Erable, Matériaux \& Techniques 103 (2015) 202

[4] A. Simons, A. Laborel-Préneron, A. Bertron, et al., Matériaux \& Techniques 103 (2015) 206

[5] A. Bertron, B. Erable, M. Alquier, et al., Matériaux $\&$ Techniques 101 (2013) 104

[6] C. Munzer, E. Belhaj, T. Meylheuc, A. Lecomte, F. Feugeas, Matériaux \& Techniques 103 (2015) 208

[7] S. Chakri, P. David, I. Frateur, et al., Matériaux $\&$ Techniques 103 (2015) 209

[8] C. Chagnot C. Shen, C. Munzer, et al., Matériaux \&3 Techniques 103 (2015) 205 